\title{
Two-Phase Flow in Heterogeneous Porous Media with Non-Wetting Phase Trapping
}

\author{
Adam Szymkiewicz • Rainer Helmig • \\ Hartmut Kuhnke
}

Received: 21 January 2010 / Accepted: 3 June 2010 / Published online: 24 June 2010

(C) The Author(s) 2010. This article is published with open access at Springerlink.com

\begin{abstract}
This article presents a mathematical model describing flow of two fluid phases in a heterogeneous porous medium. The medium contains disconnected inclusions embedded in the background material. The background material is characterized by higher value of the non-wetting-phase entry pressure than the inclusions, which causes non-standard behavior of the medium at the macroscopic scale. During the displacement of the non-wetting fluid by the wetting one, some portions of the non-wetting fluid become trapped in the inclusions. On the other hand, if the medium is initially saturated with the wetting phase, it starts to drain only after the capillary pressure exceeds the entry pressure of the background material. These effects cannot be represented by standard upscaling approaches based on the assumption of local equilibrium of the capillary pressure. We propose a relevant modification of the upscaled model obtained by asymptotic homogenization. The modification concerns the form of flow equations and the calculation of the effective hydraulic functions. This approach is illustrated with two numerical examples concerning oil-water and $\mathrm{CO}_{2}$-brine flow, respectively.
\end{abstract}

Keywords Two-phase flow modeling $\cdot$ Capillary trapping $\cdot$ Upscaling $\cdot$ Homogenization

\section{Introduction}

Modeling of two-phase flow in porous media is required in many environmental engineering applications. Whenever two fluids are present in a porous medium, one of them shows greater affinity to the solid skeleton and tends to adhere to its surface. It is called the wetting fluid, while the other one-non-wetting. In natural porous media, the wetting fluid is usually water,

\footnotetext{
A. Szymkiewicz $(\varangle)$

Department of Geotechnics, Geology and Marine Engineering, Faculty of Civil and Environmental Engineering, Gdańsk University of Technology, ul. Narutowicza 11/12, 80-233 Gdańsk, Poland e-mail: adams@pg.gda.pl

R. Helmig · H. Kuhnke

Department of Hydromechanics and Modeling of Hydrosystems, Institute of Hydraulic Engineering, Universität Stuttgart, Pfaffenwaldring 61, 70550 Stuttgart, Germany
} 
while the non-wetting one can be, for example, air or oil. When the wetting fluid invades pore space occupied by a non-wetting fluid, it moves in the form of film along the walls of the pores. As the amount of the wetting fluid increases, the film becomes thicker. Consequently, the non-wetting fluid is displaced at first from the pores of finest diameter and later from the larger ones. Owing to the action of the adhesive forces on the wetting fluid and the presence of the surface tension between the fluid phases the pressure in the wetting fluid is lower than in the non-wetting one. The difference is known as the capillary pressure. The value of the capillary pressure decreases as the wetting fluid saturation increases. The relation between the capillary pressure and the wetting fluid content (or saturation) is known as the capillary or retention function.

A characteristic feature of many porous materials is the presence of a distinct entry pressure. It means that when the medium is fully saturated with the wetting fluid, the non-wetting phase can invade the pore space only when the difference between the non-wetting and wetting phase pressures exceeds a specific positive value. The actual value of the entry pressure depends on the pore diameter and is higher in finetextured materials. Natural porous media often contain lenses or inclusions of coarser texture than the surrounding material (background). During capillary-dominated infiltration of the wetting fluid, the background material becomes saturated faster than inclusions. If the capillary pressure in inclusions does not exceed the entry pressure of the background, then the non-wetting fluid cannot leave the inclusions and becomes immobilized (trapped). Consequently, it is impossible to fully saturate the medium with the wetting fluid and the effective wetting phase conductivity is reduced because of the presence of unsaturated regions. The non-wetting phase trapping caused by material heterogeneity with respect to the entry pressure, which is the subject of this article, should not be confused with other phase-trapping mechanisms. For example, wetting phase can be trapped in fine-textured material surrounded by coarse-textured material, if the capillary pressure in the latter one becomes so high, that the wetting phase permeability is reduced to zero (Quintard and Whitaker 1988). Both mentioned processes result from the heterogeneity of the medium at large (macroscopic) scale, much larger than the pore scale. On the other hand, phase trapping can be also observed at the pore scale, where small portions of fluid become immobilized due to complex geometry of the pores. Pore-scale trapping is not the subject of this study.

The characteristic size of material heterogeneities in natural porous medium is usually much smaller than the scale of practical interest; thus, explicit representation of the heterogeneous structure in the numerical solution is hardly possible. Instead, macroscopic models are used, in which the medium is characterized by "average" ("effective") properties. These properties have to be estimated from the local-scale description of the medium by means of an upscaling procedure. Upscaling received much attention in the literature, especially in the context of petroleum engineering (e.g., Barker and Thibeau 1997; Ekrann and Aasen 2000; Virnovsky et al. 2004) and subsurface hydrology (e.g., Eichel et al. 2005; Vereecken et al. 2007). An emerging field of application of upscaling techniques is the geological sequestration of $\mathrm{CO}_{2}$, considered to be one of the possible means to mitigate global climate changes caused by human activity (e.g., Kopp et al. 2009a,b).

Two-phase flow in porous media can be driven by viscous, gravitational and capillary forces (Stephen et al. 2001; Jonoud and Jackson 2008). Their relative importance influences the choice of appropriate upscaling method. In this article we focus on the case of flow dominated by capillary forces at the length scale corresponding to the size of the heterogeneities. This allows us to use macroscopic models based on the assumption of local capillary equilibrium. A number of such models can be found in the literature (e.g., Saez et al. 1989; Quintard and Whitaker 1988; Braun et al. 2005; Lewandowska and 
Laurent 2001). However, most of them assume that the pressures in both fluid phases are continuous across the material interfaces, which makes them unsuitable for the description of the trapping effects. Non-wetting phase trapping was investigated in the framework of the homogenization theory by van Duijn et al. $(2002,2007)$ but their work was limited to one-dimensional layered medium and horizontal incompressible flow.

The objective of this study is to propose a macroscopic model for two-phase flow in heterogeneous medium which accounts for the trapping effects and can be applied for an arbitrary geometry of inclusions and arbitrary flow direction. We start our presentation with the standard model obtained by homogenization, which does not include trapping. Next, the proposed modifications of the model are discussed. Numerical examples are presented to show the influence of trapping on the effective hydraulic functions and on the macroscopic behavior of the medium.

\section{Mathematical Formulation of Two-Phase Flow}

The flow of two slightly compressible immiscible fluids in a rigid porous medium is described by two coupled partial differential equations of parabolic type, each of them of the following form (e.g Saez et al. 1989):

$$
\theta_{\alpha} c_{\alpha} \frac{\partial p_{\alpha}}{\partial t}+\rho_{\alpha} \frac{\partial \theta_{\alpha}}{\partial t}-\nabla \cdot\left(\rho_{\alpha} \frac{\mathbf{k}_{\alpha}}{\mu_{\alpha}} \nabla\left(p_{\alpha}+\rho_{\alpha} g x_{3}\right)\right)=0
$$

where the subscript $\alpha$ denotes the fluid phase ( $\alpha=w$ or $\alpha=n$ for the wetting and non-wetting phase, respectively), $\theta$ denote volumetric phase content, $p$-pressure, $\rho$-fluid density, $c$-fluid compressibility coefficient, $k$-phase permeability tensor, $\mu$-fluid dynamic viscosity, $g$ - gravitational acceleration, and $x_{3}$-vertical spatial direction oriented positively upward. The volumetric content of phase $\alpha$ can be expressed in terms of the medium porosity $\phi$ and fluid saturation $S$ :

$$
\theta_{\alpha}=\phi S_{\alpha}
$$

Since only two fluids are present in the pore space one can write:

$$
\theta_{w}+\theta_{n}=\phi \text { or } S_{w}+S_{n}=1
$$

It is a common approach to introduce the concept of residual saturations for each of the phases. They represent the point at which the considered phase loses its mobility, and they are related to the phenomenon of pore-scale trapping of fluids in porous media. In this article, we focus on the large-scale trapping, which can occur independently of the pore-scale processes. In order to keep the presentation general, we take into account the residual saturations related to pore scale trapping $S_{r w}$ and $S_{r n}$ and the corresponding residual phase contents $\theta_{r w}$ and $\theta_{r n}$, and we define the normalized wetting saturation $S_{e w}$ as

$$
S_{e w}=\frac{S_{w}-S_{r w}}{1-S_{r w}-S_{r n}}
$$

$S_{\text {ew }}$ is usually called effective wetting saturation. In this article, we prefer to call it "normalized", because the term "effective" is used in conjunction with the upscaled parameters.

The difference between the non-wetting and wetting pressure is known as the capillary pressure and can be defined as a function of the normalized wetting fluid saturation:

$$
p_{n}-p_{w}=p_{c}\left(S_{e w}\right)
$$


The permeability of the medium with respect to each phase can be written as

$$
\mathbf{k}_{\alpha}=\mathbf{k}_{p} k_{r \alpha}
$$

where $\mathbf{k}_{p}$ is the intrinsic permeability tensor and $k_{r \alpha}$ is the relative permeability of the phase. The relative permeability depends on the normalized wetting fluid saturation. In order to describe the relations between $p_{c}, S_{e w}, k_{r w}$ and $k_{r n}$ the analytical models of van Genuchten-Mualem (van Genuchten 1980; Mualem 1976) and Brooks-Corey-Burdine (Brooks and Corey 1964; Burdine 1953) are widely used. The latter one accounts for the entry pressure for the non-wetting phase and has the following form:

$$
\begin{aligned}
& S_{e w}= \begin{cases}\left(\frac{p_{c}}{p_{e}}\right)^{-\lambda} & \text { if } p_{c}>p_{e} \\
1 & \text { if } p_{c} \leq p_{e}\end{cases} \\
& k_{r w}=S_{e w}^{3+2 / \lambda} \\
& k_{r n}=\left(1-S_{e w}\right)^{2}\left(1-S_{e w}^{1+2 / \lambda}\right)
\end{aligned}
$$

where $p_{e}$ is the entry pressure, and $\lambda$ is a parameter depending on the texture of the medium. It is well known that this relation is not unique because it shows hysteresis with respect to imbibition-drainage cycles. In this article, we do not consider this effect explicitly. However, the proposed approach can be also used if the materials composing the heterogeneous medium show hysteretic behavior, as will be explained later.

\section{Upscaled Model for Two-Phase Flow with Entry Pressure Effects}

Let us consider two-phase flow in a medium composed of a continuous fine-textured background material with coarse-textured inclusions (lenses). The inclusions are not connected with each other (Fig. 1). Each of the two materials is characterized by its own set of capillary and permeability functions. We are interested in the description of flow at the scale much larger than the size of a single lens, which means that an upscaling method should be used. The approach presented here is an extension of the upscaled model obtained by asymptotic homogenization (Saez et al. 1989) to the case of flow with entry pressure effects. The homogenization method has the advantage of mathematical rigorousness and allows us to establish explicit connections between processes at various scales. The model is based on the following assumptions:

Fig. 1 Structure of a periodic heterogeneous medium

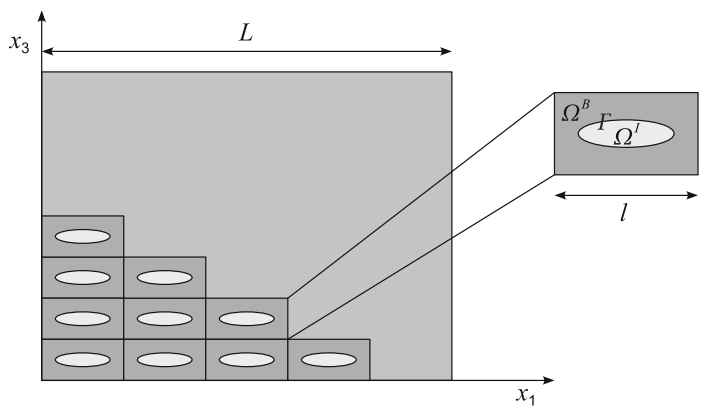


- The medium has periodic structure as shown in Fig. 1, where $\Omega$ denotes the periodic cell, and $\Omega^{B}$ and $\Omega^{B}$ the parts of the cell occupied by background material and inclusions, respectively; the interface separating the two regions is denoted by $\Gamma$.

- The medium has two characteristic lengths: the macroscopic length $L$ and the microscopic length $l$; their ratio is the scale parameter, which should be small, $\varepsilon=l / L \ll 1$.

- The fluid flow within each of the two regions is described by Eq. 1:

$-\quad$ in $\Omega^{B}$ :

$$
\begin{aligned}
\theta_{w}^{B} c_{w} \frac{\partial p_{w}^{B}}{\partial t}+\rho_{w}^{B} \frac{\partial \theta_{w}^{B}}{\partial t}-\nabla \cdot\left(\rho_{w} \frac{\mathbf{k}_{w}^{B}}{\mu_{w}} \nabla\left(p_{w}^{B}+\rho_{w} g x_{3}\right)\right) & =0 \\
\theta_{n}^{B} c_{n} \frac{\partial p_{n}^{B}}{\partial t}+\rho_{n}^{B} \frac{\partial \theta_{n}^{B}}{\partial t}-\nabla \cdot\left(\rho_{n} \frac{\mathbf{k}_{n}^{B}}{\mu_{n}} \nabla\left(p_{n}^{B}+\rho_{n} g x_{3}\right)\right) & =0
\end{aligned}
$$

$-\quad$ in $\Omega^{I}$ :

$$
\begin{aligned}
\theta_{w}^{I} c_{w} \frac{\partial p_{w}^{I}}{\partial t}+\rho_{w}^{I} \frac{\partial \theta_{w}^{I}}{\partial t}-\nabla \cdot\left(\rho_{w} \frac{\mathbf{k}_{w}^{I}}{\mu_{w}} \nabla\left(p_{w}^{I}+\rho_{w} g x_{3}\right)\right) & =0 \\
\theta_{n}^{I} c_{n} \frac{\partial p_{n}^{I}}{\partial t}+\rho_{n}^{I} \frac{\partial \theta_{n}^{I}}{\partial t}-\nabla \cdot\left(\rho_{n} \frac{\mathbf{k}_{n}^{I}}{\mu_{n}} \nabla\left(p_{n}^{I}+\rho_{n} g x_{3}\right)\right) & =0
\end{aligned}
$$

- The characteristic time of the process corresponds to the time of flow at the macroscopic scale.

- The permeabilities of the two materials are of the same order of magnitude.

- The capillary forces dominate over the viscous and gravitational forces at the scale of a single periodic cell.

The last condition can be quantified by two dimensionless numbers. The Bond number $N_{B}$ represents the ratio of gravitational to capillary forces, while the capillary number $N_{C}$ represents the ratio of viscous to capillary forces (e.g., Eichel et al. 2005):

$$
\begin{aligned}
& N_{B}=\frac{\Delta \rho g l_{B}}{\hat{p}_{c}} \ll 1 \\
& N_{C}=\frac{\mu_{\alpha} \hat{v}_{\alpha} l_{C}}{\hat{k} \hat{p}_{c}} \ll 1
\end{aligned}
$$

where $\Delta \rho$ is density difference between the two fluids, $l_{B}$ and $l_{C}$ - characteristic local lengths, chosen according to the flow direction, $\hat{p}_{c}$-characteristic value of the capillary pressure (usually assumed equal to the entry pressure in Brooks-Corey model), $\hat{k}$-characteristic permeability value, and $\hat{v}_{\alpha}$ - characteristic velocity of the given phase. The characteristic length for the Bond number $l_{B}$ can be assumed equal to the vertical dimension of a single heterogeneity (inclusion), while the length in the capillary number $l_{C}$ can be taken as the dimension of the heterogeneity in the direction of the flow. For a more detailed discussion of the role of dimensionless numbers in upscaling see Stephen et al. (2001) and Jonoud and Jackson (2008).

The form of the upscaled model depends on the continuity of the non-wetting fluid across the material interface $\Gamma$ between the background material and inclusions. One can distinguish three cases, which are described in the following paragraphs. 


\subsection{Case 1: $p_{c}^{B}>p_{e}^{B}$}

If the capillary pressure in the background material is above the entry pressure, then both phases are present and active in each of the two regions. Thus, we can assume that the pressures in each of the phases (and consequently also the capillary pressure) are continuous across the background-inclusion interface:

$$
\begin{array}{ll}
p_{w}^{B}=p_{w}^{I} & \text { on } \quad \Gamma \\
p_{n}^{B}=p_{n}^{I} & \text { on } \quad \Gamma
\end{array}
$$

Moreover, we assume the continuity of the normal mass fluxes across the interface:

$$
\begin{aligned}
-\rho_{w} & \frac{\mathbf{k}_{w}^{B}}{\mu_{w}} \nabla\left(p_{w}^{B}+\rho_{w}^{B} g x_{3}\right) \mathbf{n}=-\rho_{w} \frac{\mathbf{k}_{w}^{I}}{\mu_{w}} \nabla\left(p_{w}^{I}+\rho_{w}^{I} g x_{3}\right) \mathbf{n} \quad \text { on } \quad \Gamma \\
-\rho_{n} \frac{\mathbf{k}_{n}^{B}}{\mu_{n}} \nabla\left(p_{n}^{B}+\rho_{n}^{B} g x_{3}\right) \mathbf{n} & =-\rho_{n} \frac{\mathbf{k}_{n}^{I}}{\mu_{n}} \nabla\left(p_{n}^{I}+\rho_{n}^{I} g x_{3}\right) \mathbf{n} \quad \text { on } \quad \Gamma
\end{aligned}
$$

where $\mathbf{n}$ is the unit vector normal to the interface $\Gamma$. The above interface conditions were used by Saez et al. (1989) to derive homogenized model of the following form:

$$
\begin{gathered}
\theta_{w}^{E} c_{w} \frac{\partial p_{w}^{E}}{\partial t}+\rho_{w}^{E} \frac{\partial \theta_{w}^{E}}{\partial t}-\nabla \cdot\left(\rho_{w} \frac{k_{w}^{E}}{\mu_{w}} \nabla\left(p_{w}^{E}+\rho_{w}^{E} g x_{3}\right)\right)=0 \\
\theta_{n}^{E} c_{n} \frac{\partial p_{n}^{E}}{\partial t}+\rho_{n}^{E} \frac{\partial \theta_{n}^{E}}{\partial t}-\nabla \cdot\left(\rho_{n} \frac{k_{n}^{E}}{\mu_{n}} \nabla\left(p_{n}^{E}+\rho_{n}^{E} g x_{3}\right)\right)=0
\end{gathered}
$$

where the superscript $E$ denotes the effective macroscopic variables. The major result of the homogenization is that the pressures in each phase can be treated as uniform within a unit cell. The phase densities also assume uniform values corresponding to the macroscopic pressure heads, $\rho_{\alpha}^{E}=\rho_{\alpha}\left(\rho_{c}^{E}\right)$. Moreover, the capillary pressure is also uniform and can be regarded as a macroscopic variable. However, the saturations and phase contents are different in each of the two regions, because the background material and inclusions are characterized by different $p_{c}-S_{e w}$ curves. The effective (average) porosity and volumetric phase contents are defined as weighted arithmetic means:

$$
\begin{aligned}
\phi^{E} & =w^{B} \phi^{B}+w^{I} \phi^{I} \\
\theta_{w}^{E} & =w^{B} \theta_{w}^{B}\left(p_{c}^{E}\right)+w^{I} \theta_{w}^{I}\left(p_{c}^{E}\right) \\
\theta_{n}^{E} & =w^{B} \theta_{n}^{B}\left(p_{c}^{E}\right)+w^{I} \theta_{n}^{I}\left(p_{c}^{E}\right)
\end{aligned}
$$

where $w^{B}$ and $w^{I}$ are the volumetric fractions of the background material and inclusions, respectively. The values of the macroscopic phase contents change in the range defined by the macroscopic residual phase contents:

$$
\begin{aligned}
\theta_{r w}^{E} & \leq \theta_{w}^{E} \leq \phi^{E}-\theta_{r n}^{E} \\
\theta_{r n}^{E} & \leq \theta_{n}^{E} \leq \phi^{E}-\theta_{r w}^{E}
\end{aligned}
$$

The macroscopic residual phase contents are weighted arithmetic averages of the residual phase contents of the two materials:

$$
\begin{aligned}
\theta_{r w}^{E} & =w^{B} \theta_{r w}^{B}+w^{I} \theta_{r w}^{I} \\
\theta_{r n}^{E} & =w^{B} \theta_{r n}^{B}+w^{I} \theta_{r n}^{I}
\end{aligned}
$$


The macroscopic saturations of each phase can be expressed as:

$$
\begin{aligned}
& S_{w}^{E}=\theta_{w}^{E} / \phi^{E} \\
& S_{n}^{E}=\theta_{n}^{E} / \phi^{E}
\end{aligned}
$$

The effective permeability of each phase depends on the permeabilities of the two materials, on the phase saturations, and on the geometry of the unit cell (volumetric fractions of the materials and their spatial arrangement). The effective permeability function is obtained by repetitive solution of the so-called local boundary value problem defined over the periodic cell. The algorithm is as follows:

- Choose a value of the macroscopic capillary pressure $p_{c}^{E}$, assumed to be constant over the periodic cell.

- For each phase, compute the corresponding saturations in each of the materials and the upscaled saturations.

- Compute the wetting phase permeabilities in each material.

- For each of the spatial directions $i=1,2,3$, solve the following equation:

$$
-\nabla \cdot \mathbf{k}_{w}\left(\nabla \bar{b}_{i}+\nabla \tilde{b}_{i}\right)=0
$$

where $\bar{b}_{i}$ and $\tilde{b}_{i}$ represent the average values of the phase pressure and its local scale fluctuation, respectively. The components of the gradient $\nabla \bar{b}_{i}$ are set to one in the direction $i$, and zero for other directions $\left(\nabla \bar{b}_{1}=[1,0,0]^{T}\right.$, etc), so that the only unknown in Eq. 31 is $\tilde{b}_{i}$. The permeability depends on the position, i.e., $\mathbf{k}_{w}=\mathbf{k}_{p}^{B} k_{r w}^{B}\left(p_{c}\right)$ for $x^{\prime} \in \Omega^{B}$ and $\mathbf{k}_{w}=\mathbf{k}_{p}^{I} k_{r w}^{I}\left(p_{c}\right)$ for $x \in \Omega^{I}$. Note that the problem is linear, since $\mathbf{k}_{w}$ does not depend on $\tilde{b}_{i}$. Periodic boundary conditions are applied for $\tilde{b}_{i}$ along the boundaries. In order to ensure uniqueness of the solution and zero average value for $\tilde{b}_{i}$, a Dirichlet boundary condition $\tilde{b}_{i}=0$ should be specified at a single arbitrarily chosen point of the boundary. For details of computations, see, for example Szymkiewicz (2005):

- Based on the solution, compute the components of the effective permeability tensor:

$$
k_{w, i j}^{E}=\frac{\int_{\Omega} k_{w, i j}\left(\delta_{i j}+\left(\nabla \tilde{b}_{i}\right)_{j}\right) \mathrm{d} \Omega}{\int_{\Omega}\left(\delta_{i j}+\left(\nabla \tilde{b}_{i}\right)_{j}\right) \mathrm{d} \Omega}
$$

where $\left(\nabla \tilde{b}_{i}\right)_{j}$ denote the $j$-th component of the gradient of $\tilde{b}_{i}$;

- repeat the last three points for the non-wetting phase.

This procedure allows us to obtain the effective permeability tensors for each phase in function of the capillary pressure values in tabulated form. Note that it is possible to define the effective intrinsic permeability tensor $\mathbf{k}_{p}^{E}$, which is given by the solution of Eq. 31 with $k_{r w}=1$ for both materials. However, it is impossible to express the upscaled permeability as a product of $\mathbf{k}_{p}^{E}$ and a scalar relative permeability function, since the upscaled relative permeability is direction dependent, except for the case when both materials have the same relative permeability functions.

This approach for permeability upscaling was proposed by Saez et al. (1989) and Amaziane et al. (1991) as a result of asymptotic homogenization and by Quintard and Whitaker (1988) as a result of volumetric averaging procedure. It fits well into the framework of steady-state upscaling based on capillary equilibrium assumption (Braun et al. 2005; Eichel et al. 2005). 
The solution of local boundary value problem (31) for each spatial direction is actually equivalent to the solution of steady single-phase flow with periodic boundary conditions. Alternatively, renormalization or simple averaging formulas can be used to estimate the upscaled permeability from its local distribution within the unit cell $\Omega$. However, the procedure outlined above seems more accurate for complex geometries (Braun et al. 2005; Eichel et al. 2005).

\subsection{Case 2: $p_{c}^{B}<p_{e}^{B}$-Infiltration}

The model presented above can be extended to account for the effects of high entry pressure in the background material. At first, let us consider the case when a medium initially saturated with the non-wetting phase is subjected to infiltration of the wetting phase. As long as both phases are present in the background and inclusions, the standard model presented above can be used. However, when the macroscopic capillary pressure reaches the value of $p_{e}^{B}$, i.e., the entry pressure of the background material, the normalized non-wetting phase saturation in the background becomes zero (the actual non-wetting saturation is equal to the residual saturation, and the non-wetting permeability is equal to zero). The corresponding non-wetting phase content in inclusions is equal to

$$
\theta_{n}^{I_{*}}=\theta_{r n}^{I}+\left(1-S_{e w}^{I}\left(p_{e}^{B}\right)\right)\left(\phi^{I}-\theta_{r n}^{I}-\theta_{r w}^{I}\right)
$$

and the upscaled saturation is equal to

$$
\theta_{n}^{E_{*}}=w^{B} \theta_{r n}^{B}+w^{I} \theta_{n}^{I_{*}}
$$

If the model given by Eqs. 20-32 is applied, then further decrease of the capillary pressure results in the corresponding decrease of $\theta_{n}^{I}$ and $\theta_{n}^{E}$ and the increase of the wetting phase permeability, computed from the solution of the cell problem, Eq. 31.

Such approach does not take into account the fact that the non-wetting phase cannot leave the inclusions, because it cannot overcome the entry pressure in the background material. In order to include this effect, the interface conditions (16)-(19) and the upscaled equations should be modified.

Let us begin with the simplified case of incompressible flow $\left(c_{w}=c_{n}=0\right)$. The interface conditions can be specified as follows:

$$
\begin{array}{rlll}
p_{w}^{B} & =p_{w}^{I} & \text { on } & \Gamma \\
p_{c}^{I} & =p_{e}^{B} & \text { on } & \Gamma
\end{array}
$$

and:

$$
\begin{aligned}
- & \frac{\mathbf{k}_{w}^{B}}{\mu_{w}} \nabla\left(p_{w}^{B}+p_{w}^{B} g x_{3}\right) \mathbf{n}=-\frac{\mathbf{k}_{w}^{I}}{\mu_{w}} \nabla\left(p_{w}^{I}+\rho_{w}^{I} g x_{3}\right) \mathbf{n} \quad \text { on } \quad \Gamma \\
-\frac{\mathbf{k}_{n}^{I}}{\mu_{n}} \nabla\left(p_{n}^{I}+\rho_{n}^{I} g x_{3}\right) \mathbf{n} & =0 \quad \text { on } \quad \Gamma
\end{aligned}
$$

Note that the non-wetting phase pressure is undefined in the background material. With these boundary conditions, the upscaled equation for the wetting phase becomes

$$
-\nabla \cdot\left(\rho_{w} \frac{\mathbf{k}_{w}^{E_{*}}}{\mu_{w}} \nabla\left(p_{w}^{E}+\rho g x_{3}\right)\right)=0
$$


where $\mathbf{k}_{w}^{E_{*}}$ is the effective wetting phase permeability obtained from the solution of the local boundary value problem (31) with the relative permeability being equal to 1 in the background material and $k_{r w}^{I}\left(p_{e}^{B}\right)$ in the inclusions. Equation 39 actually describes steady flow, because the wetting saturation cannot increase any further and the fluids are incompressible. Since the background becomes impermeable with respect to the non-wetting phase, one has to set $\mathbf{k}_{n}^{E}=0$. Note that this result cannot be obtained from the solution of the cell problem, because the problem becomes ill-posed if the permeability in any part of the cell is equal to zero. The non-wetting fluid is incompressible, and so the saturation in the inclusions cannot change. Thus, the equation for the non-wetting phase becomes simply:

$$
\theta_{n}^{E}=\theta_{n}^{E_{*}}=\text { const }
$$

where the value of $\theta_{n}^{E_{*}}$ given by Eq. 34 can be interpreted as the macroscopic-scale residual non-wetting phase content due to entry effect. It depends on the shape of the capillary functions of the two materials and can be significantly larger than the residual non-wetting phase content resulting from the pore-scale trapping $\theta_{r n}^{E}$, given b y Eq. 28 .

If the non-wetting fluid is compressible, then the situation becomes more complicated, because even if the non-wetting fluid cannot escape from the inclusions to the background, its saturation in inclusions can change due to the compressibility. In this case, the following set of equations should be used:

$$
\begin{gathered}
\theta_{w}^{E} c_{w} \frac{\partial p_{w}^{E}}{\partial t}+\rho_{w}^{E} \frac{\partial \theta_{w}^{E}}{\partial t}-\nabla \cdot\left(\rho_{w} \frac{\mathbf{k}_{w}^{E *}}{\mu_{w}} \nabla\left(p_{w}^{E}+\rho g x_{3}\right)\right)=0 \\
\theta_{n}^{I} c_{n}\left(\frac{\partial p_{w}^{E}}{\partial t}+\frac{\partial p_{c}^{I}}{\partial t}\right)-\rho_{n}^{I} \frac{\partial \theta_{n}^{I}}{\partial t}=0
\end{gathered}
$$

Equation 42 states that the mass of non-wetting phase entrapped in inclusions remains constant. Note that the local capillary function is used instead of an upscaled one. The change in saturation is driven by the change in the macroscopic wetting phase pressure. A positive change in $p_{w}^{E}$ causes an increase of the non-wetting phase pressure in inclusions and, consequently, an increase in the density of the non-wetting fluid. In order to keep the mass constant, the increase of the density should be balanced by a decrease of the volume occupied by the fluid, which means in turn that the capillary pressure decreases. On the other hand, if $p_{w}^{E}$ decreases, then the capillary pressure in inclusions is expected to increase. It can even reach values higher than the entry pressure in the background, which enables the non-wetting fluid to move from inclusions to the background. In this case, one should switch again to the model with two continuous phases, given by Eqs. 20-32.

\subsection{Case 3: $p_{c}^{B}<p_{e}^{B}$-Drainage}

Another case arises when the medium is initially fully saturated with the wetting phase $\left(S_{\text {ew }}=1\right)$ and starts to be invaded by the non-wetting phase. Until the critical capillary pressure $p_{e}^{B}$ is reached, the only amount of non-wetting phase in the system results from the residual saturations, which can possibly be larger than zero in one or both of the materials. The upscaled equations have the following form:

$$
\begin{gathered}
\theta_{w}^{E} c_{w} \frac{\partial p_{w}^{E}}{\partial t}+\rho_{w}^{E} \frac{\partial \theta_{w}^{E}}{\partial t}-\nabla \cdot\left(\rho_{w} \frac{\mathbf{k}_{w}^{E}}{\mu_{w}} \nabla\left(p_{w}^{E}+\rho g x_{3}\right)\right)=0 \\
\theta_{n}^{E}=\theta_{r n}^{E}=\text { const }
\end{gathered}
$$


Fig. 2 Capillary functions of the background and inclusions and the effective capillary functions for infiltration and drainage

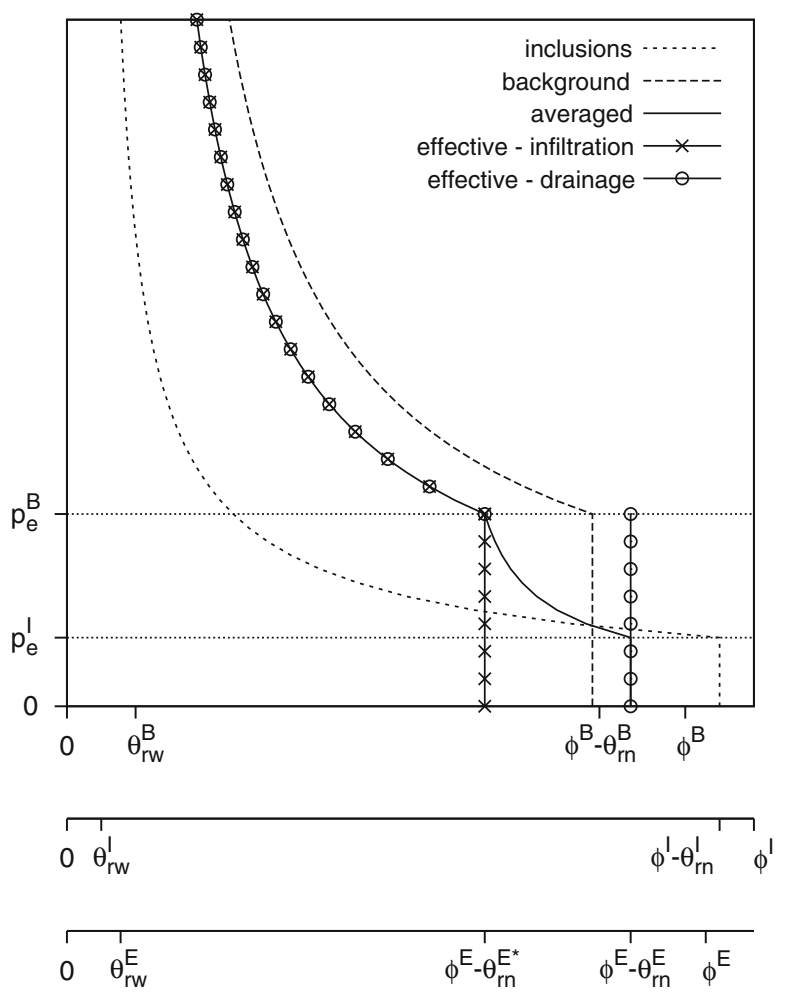

where the effective permeability of the wetting phase is equal to the effective intrinsic permeability of the medium. Note that in the above equations, we neglected the compressibility effect on the non-wetting phase at its residual saturation.

Initially, there is no capillary equilibrium between inclusions and background, because the capillary pressure in each material is equal to its entry pressure. Once the entry pressure for the matrix is exceeded, the non-wetting phase from the injection zone starts invading the system. As soon as there is a connected path of the non-wetting phase between the injection zone and inclusions, the capillary pressure in inclusions increases, until it equilibrates with the surrounding background material, with the corresponding non-wetting phase saturation in inclusions much larger than the one in the background. Thus, from the macroscopic point of view, we have a discontinuity in the effective capillary curve (Fig. 2). The saturation is constant and equal to one for $p_{c}^{E}<p_{e}^{B}$. If the capillary pressure increases by a very small value above the entry pressure of the background, then the average saturation within a unit cell sharply drops to the value corresponding to the capillary equilibrium conditions, because the inclusions desaturate quickly. Such a behavior is confirmed by physical experiments on heterogeneous media (Vasin et al. 2008); however, it is only possible, if there is initially no connected non-wetting phase in the system.

From the above considerations, we arrive at the results that in the range of capillary pressures below $p_{e}^{B}$ the medium shows hysteretic behavior, i.e., the upscaled capillary and permeability curves are different for infiltration in drainage. This hysteresis in the upscaled model results from the composite structure of the medium and is present even if each of the materials is characterized by a unique capillary curve. Such a situation is presented in Fig. 2. 
Each of the two materials is characterized by different values of porosity and the residual phase contents. The upscaled capillary functions for the infiltration and drainage are distinguished by crosses and circles, respectively. For the purposes of comparison, we also plotted the capillary curve obtained with the standard averaging procedure, Eq. 23. One can see that the three curves are identical for $p_{c}^{E}>p_{c}^{B}$. Below this value, the modified effective function can take one of two constant values, depending on the type of flow (infiltration or drainage), while the standard function is still equal to the weighted average of the phase contents for the two materials.

The approach presented here can be also used when each of the material exhibits hysteresis in its local scale capillary curve. In this case, the method of computing the effective parameters remains the same, but different local scale functions should be used as input, depending on the process which needs to be simulated.

The implications for the numerical modeling depend on the formulation used in the numerical code to solve the effective equations. Basically, two approaches are possible, i.e., either the principal variables are the pressures of the two fluids and the phase content and permeabilities are calculated as functions of the pressure difference (capillary pressure) or the principal variables are one of pressure and one of saturation, and the capillary pressure and permeabilities are calculated as the functions of the saturation. In the first case, one should remember that when the capillary pressure is below the critical value $p_{c}^{E}<p_{c}^{B}$, Eqs. 23-24 are no longer valid because the non-wetting phase content is constant and is given by Eq. 40 or 44 , depending on the process taken into consideration. In the latter case, one should remember that during infiltration, the value of the wetting phase content cannot exceed the critical value $\theta_{w}^{E_{*}}=\phi^{E}-\theta_{n}^{E_{*}}$. During the drainage, one has to deal with a discontinuous capillary function. For the purposes of consistent numerical solution, it can be approximated by a continuous capillary curve, with linear variation of $\theta_{w}^{E}$ between $p_{c}$ and $p_{c}+\delta_{p}$, where $\delta_{p}$ is a small number (similar technique for the unsaturated flow equation was used by Vasin et al. (2008).

The considerations presented above hold for the particular structure of the medium, with disconnected coarse inclusions embedded in a continuous background material. Note that if the geometry is inverse, i.e., fine inclusions are embedded in a coarse background, the standard model given by Eqs. 20-21 can be used, since the capillary pressure is always continuous within the medium. There is some qualitative similarity between the behavior of such medium with regard to the wetting phase and the behavior of the medium with coarse inclusions with respect to the non-wetting phase. For example during imbibition in the medium with fine inclusions the wetting phase tends to occupy inclusions first, while during drainage in the medium with coarse inclusions the non-wetting phase tends to occupy inclusions first. However, the effective capillary function for a medium with fine-textured inclusions does not show hysteresis.

\section{Numerical examples}

The fine scale (F) solution is obtained on a grid with explicit representation of the heterogeneous structure of the medium. It is considered as the reference, within the range of the numerical error. The upscaled (U) solution is obtained for an equivalent homogeneous domain, characterized by the effective capillary and permeability functions introduced above. It is expected to be close to the fine scale solutions, as long as the assumptions underlying the upscaling method hold. 
Both models were implemented in the $\mathrm{DuMu}^{\mathrm{X}}$ numerical framework (Flemisch et al. 2007), as modifications of its standard two-phase flow model, which is based on a fully coupled vertex-centered finite volume formulation with $p_{w}$ and $S_{n}$ as the primary variables (Helmig 1997). In the upscaled model, the values of the capillary pressure and phase permeabilities in function of the effective non-wetting saturation were interpolated linearly from a lookup table computed at the preprocessing stage. The solution of local boundary value problem, which defines the effective permeabilities, was performed with a separate numerical code, based on the cell centered finite volume discretization (see Szymkiewicz 2005).

\subsection{Example 1: Horizontal Flow in Oil-Water System}

The first example deals with horizontal displacement of oil by water. The flow is isothermal and incompressible. The densities and viscosities of the fluids are listed in Table 1. We consider a two-dimensional (2D) geometry with square-shaped inclusions, as shown in Fig. 3a. The volumetric fractions of the background material and inclusions are $w^{B}=0.51$ and $w^{I}=0.49$, respectively. Each of the two materials is characterized by Brooks-CoreyBurdine functions, Eqs. 7-9. The parameters are listed in Table 2. Initially, we assume a constant water pressure $p_{w}=101325 \mathrm{~Pa}$ and a constant capillary pressure $p_{c}=3000 \mathrm{~Pa}$ within the domain, which corresponds to the volumetric water content $\theta_{w}=0.027$ in the coarse inclusions, and $\theta_{w}=0.144$ in the fine background. At time, $t \geq 0$, at the left boundary, the water pressure is raised to $p_{w}=103325$, and full water saturation is imposed. At the opposite boundary, the initial values of $p_{w}$ and $p_{c}$ are retained. This example represents an

Table 1 Fluid properties

\begin{tabular}{lll}
\hline Fluid & Density kg m & Viscosity Pa s \\
\hline Oil & $8.90 \times 10^{2}$ & $8.00 \times 10^{-3}$ \\
Water & $1.00 \times 10^{3}$ & $1.31 \times 10^{-3}$ \\
$\mathrm{CO}_{2}$ & $(5.07 \div 5.15) \times 10^{2}$ & $(3.79 \div 3.86) \times 10^{-5}$ \\
Brine & $1.05 \times 10^{3}$ & $5.87 \times 10^{-4}$ \\
\hline
\end{tabular}

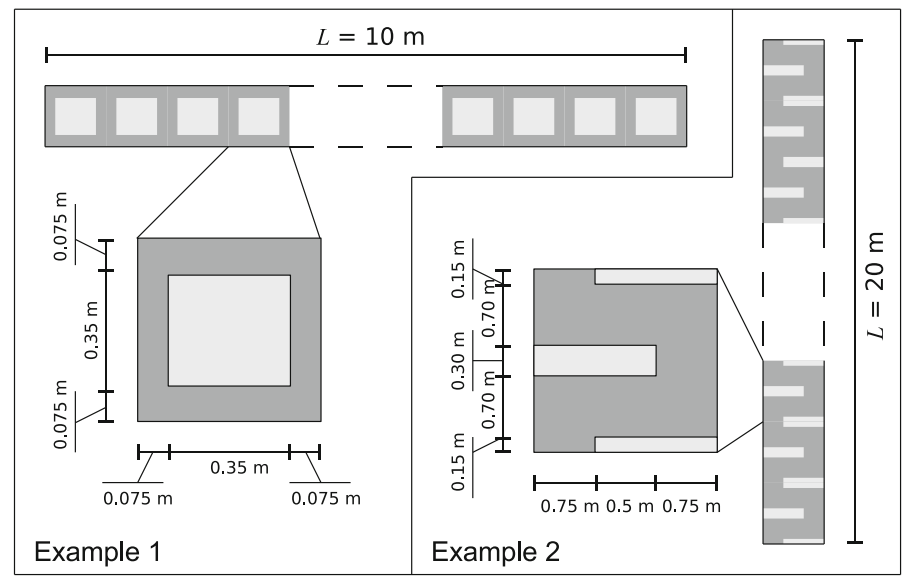

Fig. 3 Structures of porous media used in numerical examples 
Table 2 Porous media properties

\begin{tabular}{llllll}
\hline Parameter & Unit & $\begin{array}{l}\text { Example 1 } \\
\text { Background }\end{array}$ & Inclusions & $\begin{array}{l}\text { Example 2 } \\
\text { Background }\end{array}$ & Inclusions \\
\hline$\phi$ & - & 0.36 & 0.40 & 0.36 & 0.40 \\
$S_{r n}$ & - & 0.00 & 0.00 & 0.00 & 0.00 \\
$S_{r w}$ & - & 0.00 & 0.00 & 0.00 & 0.00 \\
$p_{e}$ & $\mathrm{~Pa}$ & 1400 & 500 & 14286 & 7143 \\
$\lambda$ & - & 1.2 & 1.5 & 1.2 & 1.5 \\
$k_{p}$ & $\mathrm{~m}^{2}$ & $1 \times 10^{-10}$ & $1 \times 10^{-9}$ & $1 \times 10^{-13}$ & $4 \times 10^{-13}$ \\
\hline
\end{tabular}

idealized case with capillary-dominated flow and initial capillary equilibrium. The value of capillary number $N_{C}$ defined by Eq. 15 can be estimated in the following way. The characteristic permeability and capillary pressure correspond to the parameters of the background material, the velocity is estimated as $\hat{v}_{w}=\frac{\hat{k}}{\mu_{w}} \frac{\Delta p_{w}}{L}$, where $\Delta p_{w}$ is the difference between the boundary pressures, and $L=10 \mathrm{~m}$ is the length of the domain, while the local length is $l_{C}=0.35 \mathrm{~m}$. Thus, one obtains $N_{C}=0.05$, i.e., much smaller than 1 .

The effective permeability function was obtained by repetitive solution of the local boundary value problem Eq. 31 for different values of the capillary pressure on a uniform numerical grid of 100 by 100 cells. The local and upscaled capillary and permeability functions are shown in Fig. 4. In this case, we consider only one branch of the upscaled capillary curve, corresponding to the imbibition.

The fine scale solution for this example was obtained on a uniform grid of 201 by 11 nodes, which corresponds to the spatial spacing of $\Delta x=0.05 \mathrm{~m}$. The upscaled solution was obtained on a grid of 201 by 2 nodes, i.e., the grid size in the direction parallel to the flow was the same as for the fine scale solution. In general, one could expect that the upscaled solutions are performed on much coarser grids than the fine scale solution. In this case, we preferred to use the same grid size in the direction of the flow to reduce possible influence of the numerical discretization error on the results.

The results of the simulations are presented in Figs. 5, 6, and 7. The spatial distribution of the oil saturation is presented in Fig. 5. One can see that due to the difference in the capillary functions, the oil saturation is relatively high in the inclusions, compared to the background material. The upscaled solution shows averaged oil saturation, and it matches the fine scale results well. Further confirmation can be obtained from Figs. 6 and 7, which show the distribution of the oil saturation and capillary pressure along the horizontal axis of the domain for an intermediate time and the final time. The fine scale saturation profile is highly oscillatory. The upscaled saturation values are located exactly in the middle between the fine scale values for inclusions and background, which is consistent with the fact that the volumetric fractions of the two materials are nearly the same. In the plots of the capillary pressure, it can be seen that the fine scale $p_{c}$ profile is smooth, which means that the capillary equilibrium conditions are perfectly satisfied in this case.

\subsection{Example 2: Vertical flow in $\mathrm{CO}_{2}$-brine system}

The second example shows the possible application of the presented model to the problem of $\mathrm{CO}_{2}$ sequestration. We consider a simplified setting, with quasi one-dimensional (1D) 
Fig. 4 Capillary and permeability functions used in Example 1
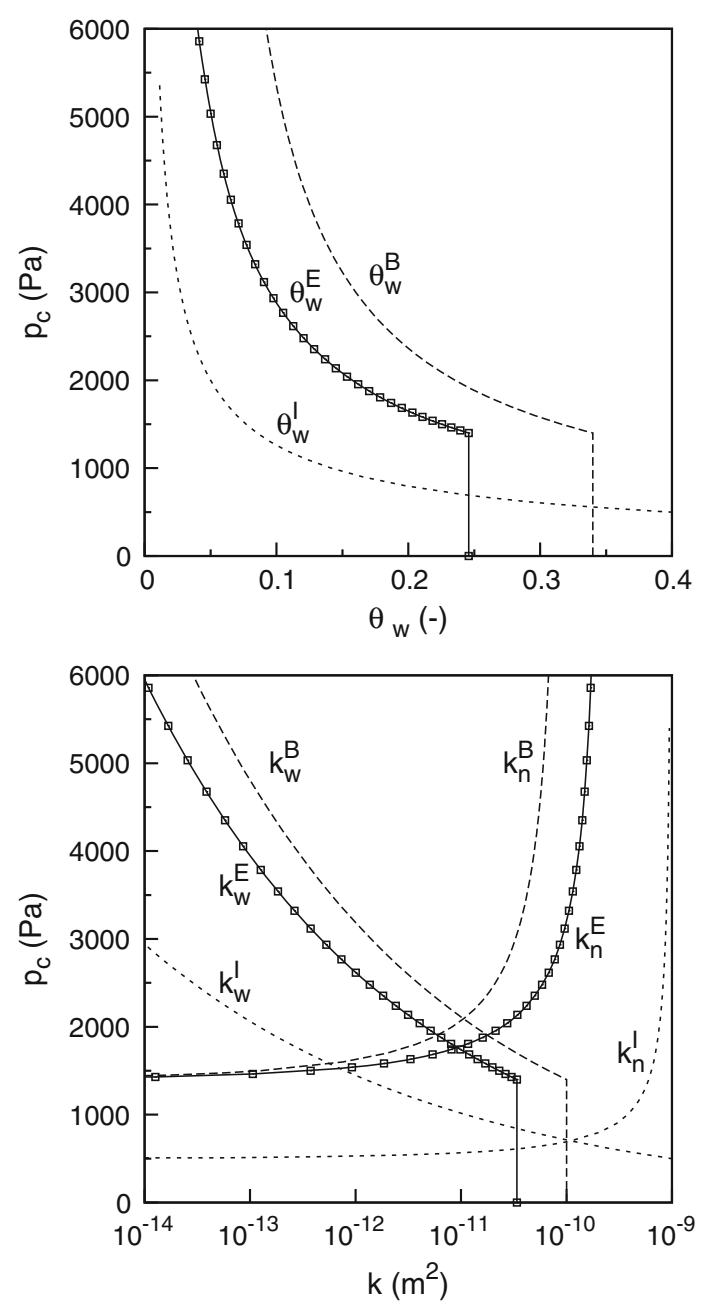

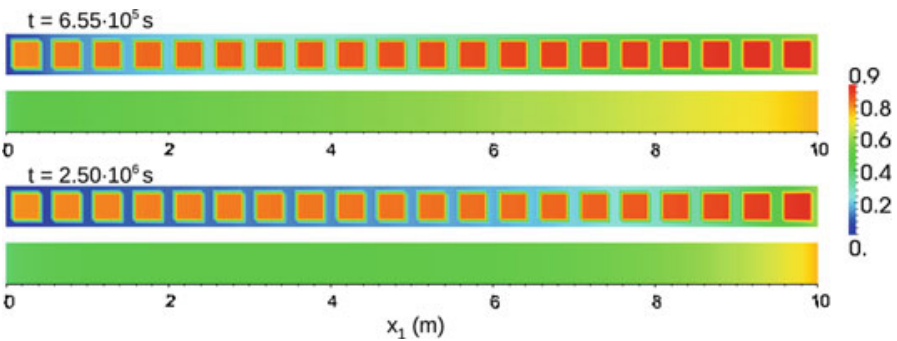

Fig. 5 Example 1: Non-wetting phase saturation distribution obtained with the fine scale model (upper graphs) and the upscaled model (lower graphs) for two selected time levels

representation of the reservoir as shown in Fig. 3. The coarse textured lenses occupy $12.5 \%$ of the volume of the medium. Moreover, we assume that each of the porous materials is characterized by a unique set of capillary and permeability curves, i.e., we neglect hysteresis 

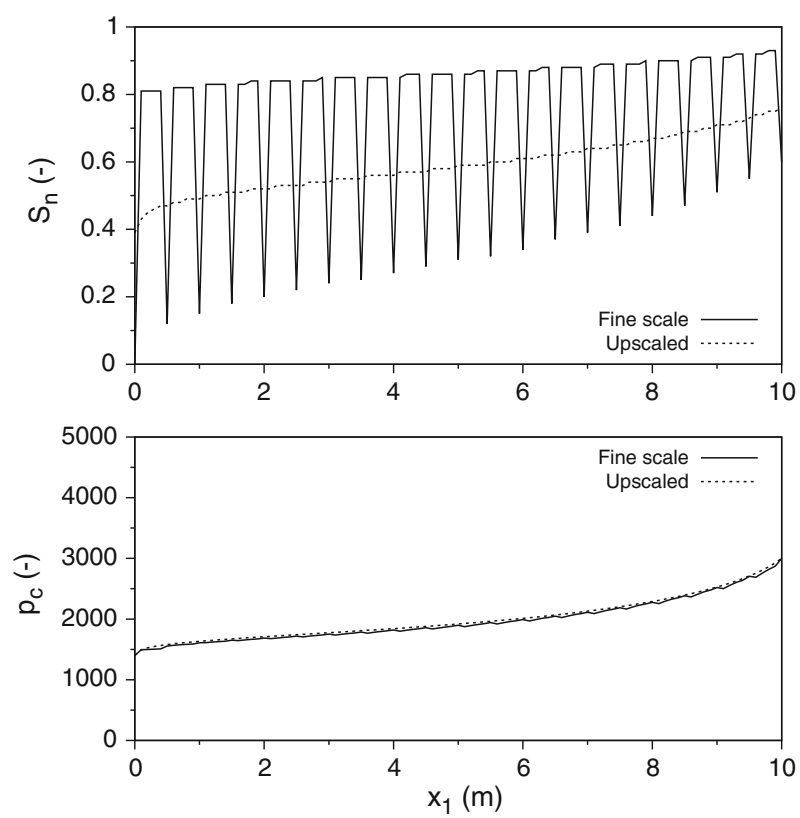

Fig. 6 Example 1: Non-wetting phase saturation and capillary pressure along the symmetry axis of the domain obtained with the fine scale model (F) and the upscaled model (U) for $t=6.55 \times 10^{5} \mathrm{~s}$
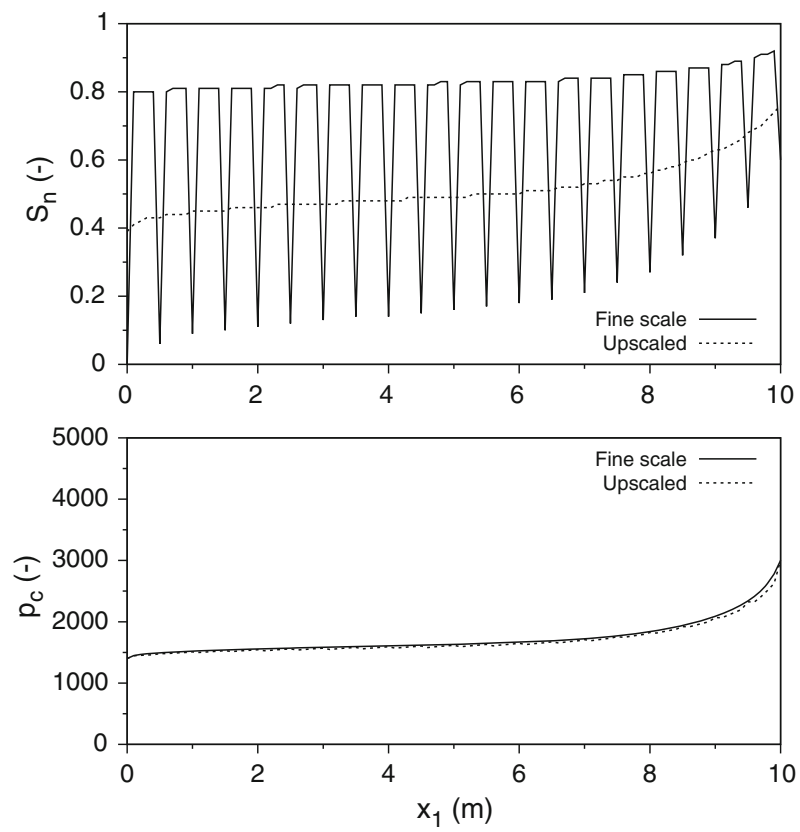

Fig. 7 Example 1: Non-wetting phase saturation and capillary pressure along the symmetry axis of the domain obtained with the fine scale model $(\mathrm{F})$ and the upscaled model $(\mathrm{U})$ for $t=2.50 \cdot 10^{6} \mathrm{~s}$ 
Fig. 8 Capillary and permeability functions used in Example 2. Empty symbols denote effective permeability in horizontal direction, solid symbols-in vertical direction
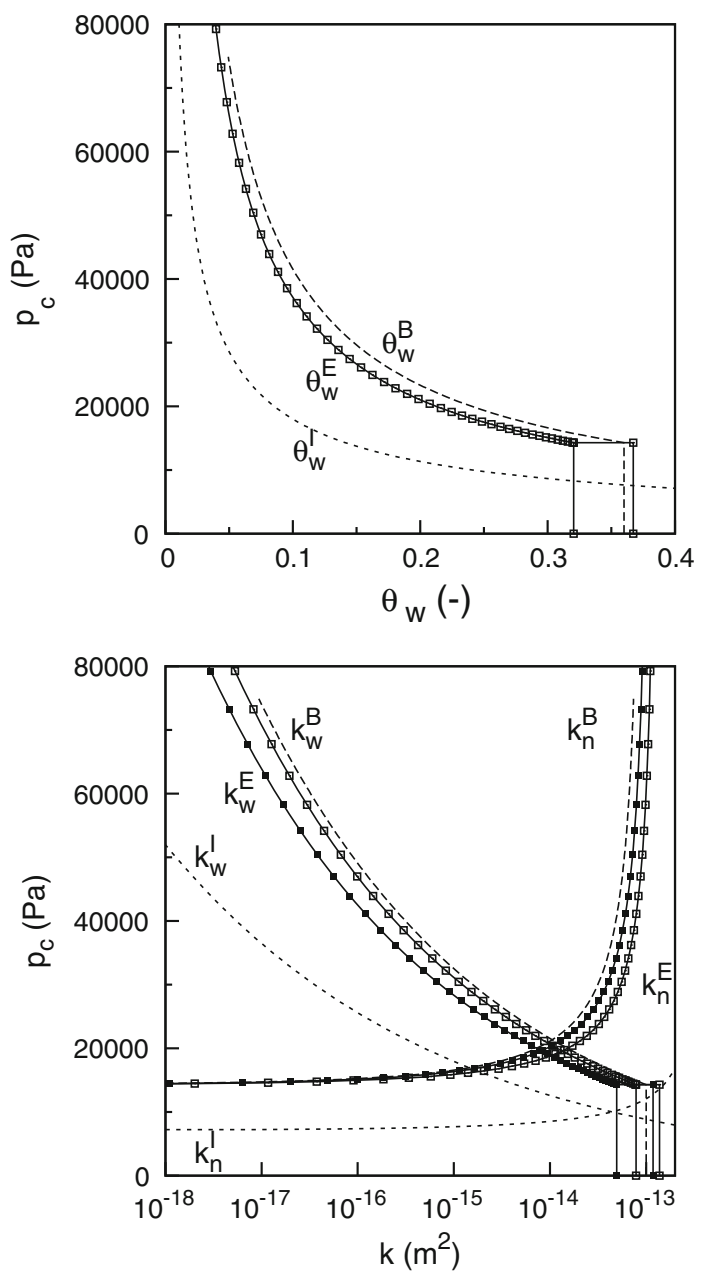

at the local scale (however, hysteresis will appear in the upscaled functions). The fluid and material properties are listed in Tables 1 and 2, respectively. In this case the wetting phase is brine, while the non-wetting phase is $\mathrm{CO}_{2}$. The density and viscosity of $\mathrm{CO}_{2}$ are computed as a function of the pressure according to the formulas of Span and Wagner (1996) (the temperature is assumed constant $T=343^{\circ} \mathrm{K}$ ). The range of entry pressures and saturations for the two porous materials is similar to the one used by Saadatpoor et al. (2009a,b). Initially the reservoir is fully saturated with brine, except for the bottom part $0 \leq x_{3} \leq 3.5 \mathrm{~m}$, where high saturation of $\mathrm{CO}_{2}$ is assumed $\left(S_{n}=0.9\right)$ as a result of a relatively fast injection. The pressure in the wetting phase (brine) is assumed constant, $p_{w}=1.5 \times 10^{7} \mathrm{~Pa}$. We neglected the initial pressure variation in the vertical direction coming from hydrostatic distribution since they are relatively small compared to the imposed pressure (about $2.0 \times 10^{5} \mathrm{~Pa}$ ). Our simulation concerns redistribution of the injected $\mathrm{CO}_{2}$, i.e., its upward flow due to buoyancy. The boundaries of the domain are impermeable, and there are no sources or sinks active for $t \geq 0$. The ratio of gravity to capillary forces can be estimated using Eq. 14 , with $\Delta \rho=500 \mathrm{~kg} / \mathrm{m}^{3}$, and $\hat{p}_{c}=14000 \mathrm{~Pa}$. Since trapping is a localized process associated with single heterogeneities, 
Fig. 9 Example 2: Non-wetting phase saturation distribution obtained with the fine scale model and the upscaled model for two selected time levels

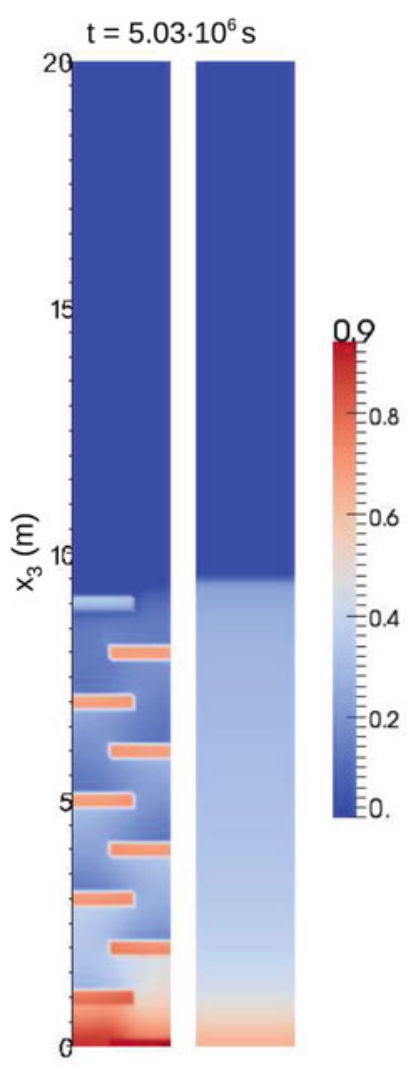

the characteristic length corresponds to the vertical dimension of a single lens, $l_{B}=30 \mathrm{~cm}$. Thus, the value of $N_{B}$ is 0.105 , indicating local dominance of the capillary forces over the gravity forces.

Note that in this case, we have both imbibition and drainage, because as $\mathrm{CO}_{2}$ moves upward, the medium is first drained (invaded by non-wetting fluid) and then imbibed again with brine. Thus, one has to account for the hysteresis in the effective capillary function and the effective permeability function, as shown in Fig. 8. Note that the effective permeabilities are different in horizontal and vertical directions, which results from the anisotropic geometry of the medium. The calculation of the effective permeability was carried out on a numerical grid of 120 by 120 cells.

The fine scale solution was obtained on a uniform grid of 201 by 21 nodes $(\Delta x=0.1 \mathrm{~m})$, while for the upscaled solution, we used a grid of 201 by 6 nodes. The fine scale solution converged very poorly and needed $220000 \mathrm{~s}$ of wall-clock time to reach the final simulation time of $10^{8} \mathrm{~s}$. In contrast, the upscaled solution required only about $500 \mathrm{~s}$ on the same machine (4 Pentium Xeon 3.00 GHz processors, 4 GB RAM).

The results obtained with both models are compared in Figs. 9, 10, and 11. Figure 9 shows the distribution of $\mathrm{CO}_{2}$ saturation at times $t=5.03 \times 10^{6} \mathrm{~s}$ and $t=1.00 \times 10^{8} \mathrm{~s}$ (the final time). It can be seen that the two solutions are in qualitative agreement. The fine scale simulation shows $\mathrm{CO}_{2}$ trapped in the coarse-textured lenses, while the rest of it occupies the upper part of the reservoir. The trapping effect is represented in the upscaled solution by 

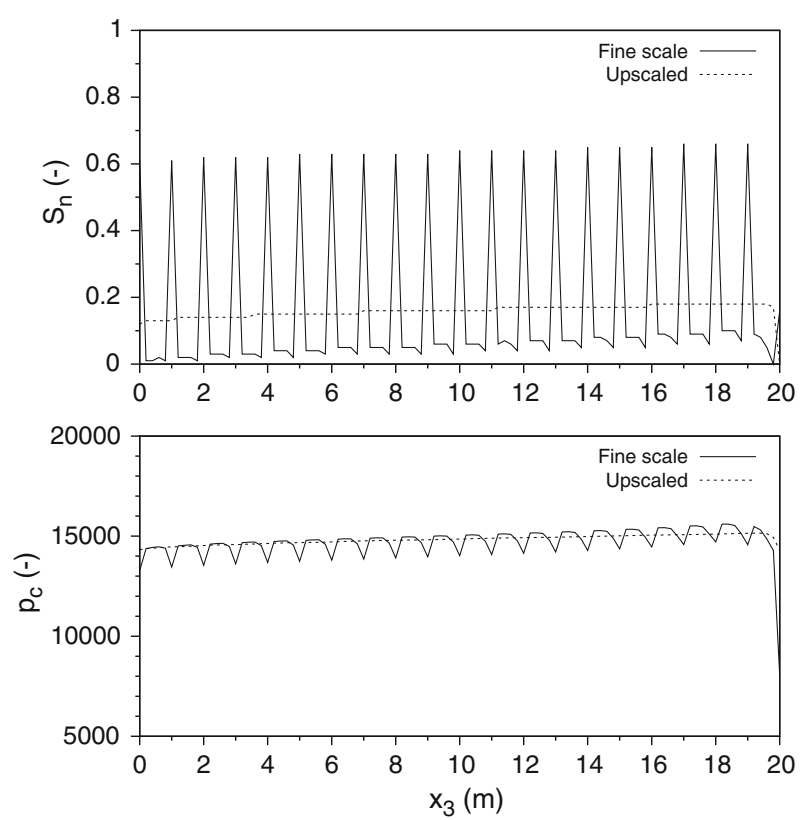

Fig. 10 Example 2: Non-wetting phase saturation and capillary pressure along the symmetry axis of the domain obtained with the fine scale model (F), and the upscaled model (U) for $t=2.00 \cdot 10^{7} \mathrm{~s}$

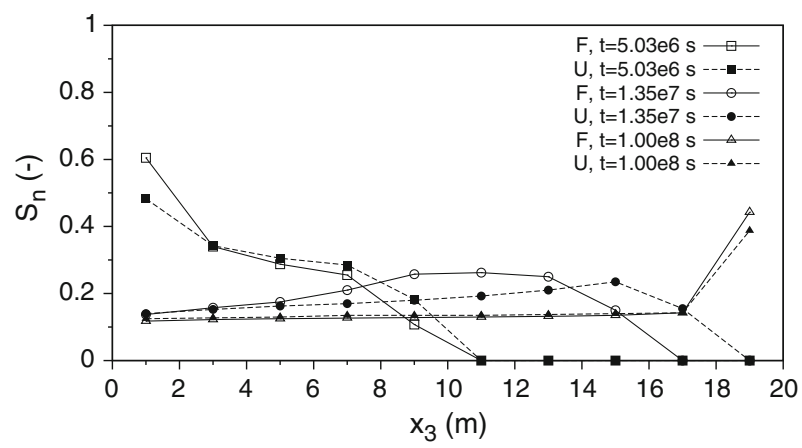

Fig. 11 Example 2: Average non-wetting phase saturation obtained with the fine scale model (F), and the upscaled model (U)

the values of $S_{n}$ larger than zero in the lower part of the domain. The two solutions also agree with respect to the time of the arrival of $\mathrm{CO}_{2}$ flume at the upper boundary of the reservoir, which is about $2.0 \times 10^{7} \mathrm{~s}$. This can be seen in Fig. 10, where the values of $S_{n}$ and $p_{c}$ along the vertical central axis of the domain are plotted. Similar to Example 1, we can see large oscillations of the saturation. The capillary pressure also shows some oscillations, but they are relatively small.

In order to allow for a quantitative comparison, we also show the values of average saturations obtained with both models, Fig. 11. The averages were taken over each of the 10 periodic cells which constitute the solution domain. It can be seen that there is some 
discrepancy between the fine scale and upscaled results for the intermediate time $t=1.35 \times$ $10^{7}$, while for the earlier and later times, the agreement is very good. Actually, for this example-in contrast to the previous one-we could expect a less than perfect agreement, due to worse separation of scales and the presence of advection terms related to the gravity. The fluid configuration in the background material does not reach full equilibrium, as can be seen in Fig. 10. Nevertheless, the upscaled model seems to produce good results also in this more difficult case.

\section{Summary and Conclusions}

An upscaling approach for two-phase flow in porous media accounting for large scale trapping of the non-wetting phase is proposed. The model is valid for capillary-dominated flow. The upscaled capillary and permeability functions are different for imbibition and drainage, showing hysteresis. This effect comes solely from the presence of material heterogeneities within the considered medium, and is not related to the hysteresis observed in homogeneous medium, which comes from the pore-scale processes.

While capillary flow is often associated with small-scale processes, it has been shown that the model can be used for large-scale applications related to $\mathrm{CO}_{2}$ storage in deep geological formations. The numerical simulations described this study indicated the importance of $\mathrm{CO}_{2}$ trapping in coarse textured inclusions and confirmed the necessity to include capillary effects in the applied simulators (Saadatpoor et al. 2009a,b). Application of the model for real problems is limited by the assumptions of capillary equilibrium. It means that the model can describe flow in geological formations with small lenses having vertical dimension of the order of a few centimeters or decimeters, for which the capillary forces dominate over gravity. If viscous and/or gravity forces play important role, then different upscaling methods should be used (e.g., Ekrann and Aasen 2000; Virnovsky et al. 2004). Information on the connectivity of the coarse-textured heterogeneities is also important, since trapping can occur only in disconnected inclusions. On the other hand, the presented model can be further developed to give a more realistic description of the processes related to the $\mathrm{CO}_{2}$ storage. This can be achieved by switching to the two-phase two-component description of gas flow and including hysteresis in the small scale capillary functions for inclusions and background.

The model described above can be also used for flow in the vadose zone, where the size of heterogeneities is often small, while capillary forces in the air-water system are significant. This process is commonly described with the Richards' equation, which does not account explicitly for the air phase, and thus may be unsuitable for soils characterized by large capillary heterogeneities, for example, if air trapping in coarse-textured inclusions is expected.

Acknowledgements The author A.S. would like to thank the German Research Foundation (DFG) for providing the financial support for the project within the Cluster of Excellence in Simulation Technology (EXC 310/1) at the University of Stuttgart. In addition, support from the International Research Training Group "Nonlinearities and Upscaling in Porous Media" (NUPUS) for the same author is kindly acknowledged here. The authors would like to thank Andreas Lauser for help in numerical implementation of the model, and two anonymous reviewers for their helpful suggestions.

Open Access This article is distributed under the terms of the Creative Commons Attribution Noncommercial License which permits any noncommercial use, distribution, and reproduction in any medium, provided the original author(s) and source are credited. 


\section{References}

Amaziane, B., Bourgeat, A., Koebbe, J.: Numerical simulation and homogenization of two-phase flow in heterogeneous porous media. Transport Porous Med. 6(5-6), 519-547 (1991)

Barker, J., Thibeau, S.: A critical review of the use of pseudo relative permeabilities for upscaling. SPE Reservoir Eng. 12(2), 138-143 (1997)

Braun, C., Helmig, R., Manthey, S.: Macro-scale effective constitutive relationships for two phase flow processes in heterogeneous porous media with emphasis on the relative permeability-saturation relationship. J. Contam. Hydrol. 76(1-2), 47-85 (2005)

Brooks, R., Corey, A.: Hydraulic Properties of Porous Media. Hydrology Paper 3. Colorado State University, Fort Collins, CO

Burdine, N.: Relative permeability calculations from pore size distribution data. Trans. Am. Inst. Min. Metall. Petrol. Eng. 198, 71-77 (1953)

Eichel, H., Helmig, R., Neuweiler, I., Cirpka, O.: Upscaling of two-phase flow processes in porous media. In: Das, D., Hassanizadeh, S. (eds.) Upscaling Multiphase Flow in Porous Media, pp. 237-257. Springer, Dordrecht (2005)

Ekrann, S., Aasen, J.: Steady-state upscaling. Transport Porous Med. 41(3), 245-262 (2000)

Flemisch, B., Fritz, J., Helmig, R., Niessner, J., Wohlmuth, B.: DUMUX: a multiscale multi-physics toolbox for flow and transport processes in porous media. In: Ibrahimbegovic, A., Dias, F., Matthies, H., Wriggers, P. (eds.) ECCOMAS Thematic Conference on Multi-scale Computational Methods for Solids and Fluids. Cachan, France, November 28-30 (2007)

Helmig, R.: Multiphase Flow and Transport Processes in the Subsurface: A Contribution to the Modeling of the Hydrosystems. Springer, Berlin (1997)

Jonoud, S., Jackson, M.: New criteria for the validity of steady-state upscaling. Transport Porous Med. 71(1), 53-73 (2008)

Kopp, A., Class, H., Helmig, R.: Investigations on $\mathrm{CO}_{2}$ storage capacity in saline aquifers-Part 1: dimensional analysis of flow processes and reservoir characteristics. Int. J. Greenhouse Gas Control 3(3), 263-276 (2009a). doi:10.1016/j.ijggc.2008.10.002

Kopp, A., Class, H., Helmig, R.: Investigations on $\mathrm{CO}_{2}$ storage capacity in saline aquifers-Part 1: estimation of storage capacity coefficients. Int. J. Greenhouse Gas Control 3(3), 277-287 (2009b). doi:10.1016/j. ijggc.2008.10.001

Lewandowska, J., Laurent, J.P.: Homogenization modeling and parametric study of moisture transfer in an unsaturated heterogeneous porous medium. Transport Porous Med. 45(3), 321-345 (2001)

Mualem, Y.: A new model for predicting the hydraulic conductivity of unsaturated porous media. Water Resour. Res. 12(3), 513-522 (1976). doi:10.1029/WR012i003p00513

Quintard, M., Whitaker, S.: Two-phase flow in heterogeneous porous media: the method of large scale averaging. Transport Porous Med. 3(4), 357-413 (1988)

Saadatpoor, E., Bryant, S., Sepehrnoori, K.: Effect of capillary heterogeneity on buoyant plumes: a new local trapping mechanism. Energy Procedia 1, 3299-3306 (2009a). doi:10.1016/j.egypro.2009.02.116

Saadatpoor, E., Bryant, S., Sepehrnoori, K.: New trapping mechanism in carbon sequestration. Transport Porous Med. (2009b). doi:10.1007/s11242-009-9446-6

Saez, A., Otero, C., Rusinek, I.: The effective homogeneous behavior of heterogeneous porous media. Transport Porous Med. 4(3), 213-238 (1989)

Span, R., Wagner, W.: A new equation of state for carbon dioxide covering the fluid region from the triple point temperature to $1100 \mathrm{~K}$ at pressures up to $800 \mathrm{MPa}$. J. Phys. Chem. Ref. Data 25, 1509-1596 (1996)

Stephen, K., Pickup, G., Sorbie, K.: The local analysis of changing force balances in immiscible incompressible twophase flow. Transport Porous Med. 45(1), 63-88 (2001)

Szymkiewicz, A.: Calculating effective conductivity of heterogeneous soils by homogenization. Arch. HydroEng. Environ. Mech. 52(2), 111-130 (2005)

van Duijn, C., Mikelic, A., Pop, I.: Effective equations for two phase flow with trapping on the micro scale. SIAM J. Appl. Math. 62, 531-1568 (2002)

van Duijn, C., Eichel, H., Helmig, R., Pop, I.: Effective equations for two-phase flow in porous media: the effect of trapping at the micro-scale. Transport Porous Med. 69(3), 411-428 (2007). doi:10.1007/ s11242-006-9089-9

van Genuchten, M.: A closed form equation for predicting the hydraulic conductivity of unsaturated soils. Soil Sci. Soc. Am. J. 44(5), 892-898 (1980)

Vasin, M., Lehmann, P., Kaestner, A., Hassanein, R., Nowak, W., Helmig, R., Neuweiler, I.: Drainage in heterogeneous sand columns with different geometric structure. Adv. Water Res. 31(9), 1205-1220 (2008) 
Vereecken, H., Kasteel, R., Vanderborght, J., Harter, T.: Upscaling hydraulic properties and soil water flow processes in heterogeneous soils: a review. Vadose Zone J. 6(1), 1-28 (2007)

Virnovsky, G., Friis, H., Lohne, A.: A steady-state upscaling approach for immiscible two-phase flow. Transport Porous Med. 54(2), 167-192 (2004) 